\title{
Weak and strong convergence theorems of modified SP-iterations for generalized asymptotically quasi-nonexpansive mappings
}

\author{
G.S. SALUJA
}

\begin{abstract}
The aim of this paper is to establish some strong and weak convergence theorems of modified SP-iterations for three generalized asymptotically quasi-nonexpansive mappings in the framework of Banach spaces. Our results extend and generalize several results from the current existing literature.
\end{abstract}

\section{INTRODUCTION}

Let $E$ be a Banach space and let $C$ be a nonempty subset $E$. We denote the set of all fixed points of $T$ by $F(T)$. The set of common fixed points of three mappings $T_{1}, T_{2}$ and $T_{3}$ will be denoted by $F=\cap_{i=1}^{3} F\left(T_{i}\right)$. Let $T: C \rightarrow C$ be a nonlinear mapping.

Definition 1.1. A mapping $T: C \rightarrow C$ is said to be:

(i) nonexpansive if

$$
\|T x-T y\| \leq\|x-y\|, \quad \forall x, y \in C ;
$$

(ii) quasi-nonexpansive if

$$
\|T x-p\| \leq\|x-p\|, \quad \forall x \in C, p \in F(T) ;
$$

(iii) asymptotically nonexpansive [4] if there exists a sequence $\left\{r_{n}\right\}$ in $[0, \infty)$ with $\lim _{n \rightarrow \infty} r_{n}=0$ such that

$$
\left\|T^{n} x-T^{n} y\right\| \leq\left(1+r_{n}\right)\|x-y\|, \quad \forall x, y \in C, n \in \mathbf{N} ;
$$

(iv) asymptotically quasi-nonexpansive [9] if $F(T) \neq \emptyset$ and there exists a sequence $\left\{r_{n}\right\}$ in $[0, \infty)$ with $\lim _{n \rightarrow \infty} r_{n}=0$ such that

$$
\left\|T^{n} x-p\right\| \leq\left(1+r_{n}\right)\|x-p\|, \quad \forall x \in C, p \in F(T), n \in \mathbf{N} ;
$$

2000 Mathematics Subject Classification. 47H09, 47H10, 47J25.

Key words and phrases. Generalized asymptotically quasi-nonexpansive mapping, modified SP-iteration process, common fixed point, Banach space, strong convergence, weak convergence. 
(v) generalized asymptotically quasi-nonexpansive [8, 11] if there exist two sequences $\left\{r_{n}\right\}$ and $\left\{s_{n}\right\}$ in $[0, \infty)$ with $\lim _{n \rightarrow \infty} r_{n}=0$ and $\lim _{n \rightarrow \infty} s_{n}=0$ such that

$$
\left\|T^{n} x-p\right\| \leq\left(1+r_{n}\right)\|x-p\|+s_{n}\left\|x-T^{n} x\right\|,
$$

for all $x \in C, p \in F(T)$ and $n \in \mathbf{N}$.

From above definitions we have the following implications: let

$C_{1}=\{T: T: C \rightarrow C$ is a nonexpansive mapping $\}$,

$C_{2}=\{T: T: C \rightarrow C$ is a quasi-nonexpansive mapping $\}$,

$C_{3}=\{T: T: C \rightarrow C$ is an asymptotically nonexpansive mapping $\}$,

(6) $C_{4}=\{T: T: C \rightarrow C$ is an asymptotically

quasi-nonexpansive mapping\},

$C_{5}=\{T: T: C \rightarrow C$ is generalized asymptotically

quasi-nonexpansive mapping\},

then, we have the following

$$
\begin{aligned}
C_{1} & \Rightarrow C_{2} . \\
C_{1} \Rightarrow C_{3} \Rightarrow C_{4} & \Rightarrow C_{5} .
\end{aligned}
$$

Example 1.1. Let $E=\mathbb{R}$ and let $T$ be defined by

$$
T(x)=\left\{\begin{array}{cl}
\frac{x}{2} \cos \frac{1}{x}, & \text { if } x \neq 0 \\
0, & \text { if } x=0
\end{array}\right.
$$

If $x \neq 0$ and $T x=x$, then $x=\frac{x}{2} \cos \frac{1}{x}$. Thus $2=\cos \frac{1}{x}$. This is impossible. $T$ is a quasi-nonexpansive mapping since if $x \in E$ and $z=0$, then

$$
\|T x-z\|=\|T x-0\|=\left|\frac{x}{2}\right|\left|\cos \frac{1}{x}\right| \leq \frac{|x|}{2}<|x|=|x-z|=\|x-z\|,
$$

and hence $T$ is an asymptotically quasi-nonexpansive mapping with constant sequence $\left\{k_{n}\right\}=\{1\}$. Hence by implication relation (7), $T$ is generalized asymptotically quasi-nonexpansive mapping. But it is not a nonexpansive mapping as $\|T x-T y\| \leq\|x-y\|$ is not satisfied for $x=\frac{2}{3 \pi}$ and $y=\frac{1}{\pi}$ and hence is not asymptotically nonexpansive mapping. Thus the class of generalized asymptotically quasi-nonexpansive mappings is larger than the class of nonexpansive, quasi-nonexpansive, asymptotically nonexpansive and asymptotically quasi-nonexpansive mappings.

(1) Modified S-iteration [1]: In 2007, Agarwal et al. [1] introduced the following iteration scheme:

$$
\begin{aligned}
x_{1} & =x \in C, \\
x_{n+1} & =\left(1-\alpha_{n}\right) T^{n} x_{n}+\alpha_{n} T^{n} y_{n}, \\
y_{n} & =\left(1-\beta_{n}\right) x_{n}+\beta_{n} T^{n} x_{n}, n \geq 1
\end{aligned}
$$


where $\left\{\alpha_{n}\right\}$ and $\left\{\beta_{n}\right\}$ are sequences in $(0,1)$ and they established some weak convergence theorems under additional conditions for nearly asymptotically nonexpansive self mappings in the framework of uniformly convex Banach spaces.

(2) Noor iteration [12]: Chose $x_{1} \in C$ and define

$$
\begin{aligned}
z_{n} & =\left(1-\gamma_{n}\right) x_{n}+\gamma_{n} T x_{n} \\
y_{n} & =\left(1-\beta_{n}\right) x_{n}+\beta_{n} T z_{n} \\
x_{n+1} & =\left(1-\alpha_{n}\right) x_{n}+\alpha_{n} T y_{n}, n \geq 1,
\end{aligned}
$$

where $\left\{\alpha_{n}\right\},\left\{\beta_{n}\right\}$ and $\left\{\gamma_{n}\right\}$ are sequences in $[0,1]$.

(3) Modified Noor iteration [26]: Chose $x_{1} \in C$ and define

$$
\begin{aligned}
z_{n} & =\left(1-\gamma_{n}\right) x_{n}+\gamma_{n} T^{n} x_{n} \\
y_{n} & =\left(1-\beta_{n}\right) x_{n}+\beta_{n} T^{n} z_{n} \\
x_{n+1} & =\left(1-\alpha_{n}\right) x_{n}+\alpha_{n} T^{n} y_{n}, n \geq 1,
\end{aligned}
$$

where $\left\{\alpha_{n}\right\},\left\{\beta_{n}\right\}$ and $\left\{\gamma_{n}\right\}$ are sequences in $[0,1]$.

Recently, Phuengrattana and Suantai [15] introduced the following iteration scheme.

(4) SP-iteration [15]: Chose $x_{1} \in C$ and define

$$
\begin{aligned}
z_{n} & =\left(1-\gamma_{n}\right) x_{n}+\gamma_{n} T x_{n} \\
y_{n} & =\left(1-\beta_{n}\right) z_{n}+\beta_{n} T z_{n} \\
x_{n+1} & =\left(1-\alpha_{n}\right) y_{n}+\alpha_{n} T y_{n}, n \geq 1,
\end{aligned}
$$

where $\left\{\alpha_{n}\right\},\left\{\beta_{n}\right\}$ and $\left\{\gamma_{n}\right\}$ are sequences in $[0,1]$.

Inspired and motivated by [15], we modify iteration scheme (11) for three generalized asymptotically quasi-nonexpansive self mappings of $C$ as follows:

(5) Modified SP-iteration: Chose $x_{1} \in C$ and define

$$
\begin{aligned}
z_{n} & =\left(1-\gamma_{n}\right) x_{n}+\gamma_{n} T_{3}^{n} x_{n} \\
y_{n} & =\left(1-\beta_{n}\right) z_{n}+\beta_{n} T_{2}^{n} z_{n} \\
x_{n+1} & =\left(1-\alpha_{n}\right) y_{n}+\alpha_{n} T_{1}^{n} y_{n}, n \geq 1,
\end{aligned}
$$

where $\left\{\alpha_{n}\right\},\left\{\beta_{n}\right\}$ and $\left\{\gamma_{n}\right\}$ are sequences in $[0,1]$.

Remark 1.1. If we take $T_{1}^{n}=T_{2}^{n}=T_{3}^{n}=T$ for all $n \geq 1$, then (12) reduces to the $S P$-iteration scheme (11) for generalized asymptotically quasinonexpansive self mapping of $C$. 
The three-step iterative approximation problems were studied extensively by Noor [12, 13], Glowinsky and Le Tallec [5], and Haubruge et al [6]. It has been shown [5] that three-step iterative scheme gives better numerical results than the two step and one step approximate iterations. Thus we conclude that three step scheme plays an important and significant role in solving various problems, which arise in pure and applied sciences.

Recently, many papers have appeared on the iterative approximation of fixed point and common fixed points of asymptotically quasi-nonexpansive mappings and generalized asymptotically quasi-nonexpansive mappings throu gh various iteration schemes in Banach spaces (see, e.g., [2, 7, 9, 16, 17, 18, 19, 20, 23]).

The purpose of this paper is to establish a necessary and sufficient condition for $\left\{x_{n}\right\}$ generated by (12) to converge to common fixed point for three generalized asymptotically quasi-nonexpansive mappings. Also we establish some strong convergence theorems of $\left\{x_{n}\right\}$ and the weak convergence theorems in uniformly convex Banach spaces, which satisfies the Opial property, or whose dual space has the Kadec-Klee property (KK-property). In fact, a dual space of a reflexive Banach space with a Fréchet differentiable norm or the Opial property also satisfies the Kadec-Klee property [24]. Our results extend and generalize the previous works from the current existing literature.

\section{Preliminaries}

For our main results, we shall need the following definitions and lemmas.

Let $E$ be a Banach space with its dimension greater than or equal to 2 . The modulus of convexity of $E$ is the function $\delta_{E}(\varepsilon):(0,2] \rightarrow[0,1]$ defined by

$$
\delta_{E}(\varepsilon)=\inf \left\{1-\left\|\frac{1}{2}(x+y)\right\|:\|x\|=1,\|y\|=1, \varepsilon=\|x-y\|\right\} .
$$

A Banach space $E$ is uniformly convex if and only if $\delta_{E}(\varepsilon)>0$ for all $\varepsilon \in(0,2]$.

Let $\mathcal{S}=\{x \in E:\|x\|=1\}$ and let $E^{*}$ be the dual of $E$, that is, the space of all continuous linear functionals $f$ on $E$.

Definition 2.1. (i) Opial condition: The space $E$ has Opial condition [14] if for any sequence $\left\{x_{n}\right\}$ in $E, x_{n}$ converges to $x$ weakly it follows that $\lim \sup _{n \rightarrow \infty}\left\|x_{n}-x\right\|<\lim \sup _{n \rightarrow \infty}\left\|x_{n}-y\right\|$ for all $y \in E$ with $y \neq x$. Examples of Banach spaces satisfying Opial condition are Hilbert spaces and all spaces $l^{p}(1<p<\infty)$. On the other hand, $L^{p}[0,2 \pi]$ with $1<p \neq 2$ fail to satisfy Opial condition.

(ii) A mapping $T: C \rightarrow C$ is said to be demiclosed at zero, if for any sequence $\left\{x_{n}\right\}$ in $K$, the condition $x_{n}$ converges weakly to $x \in C$ and $T x_{n}$ 
converges strongly to 0 imply $T x=0$.

(iii) A Banach space $E$ has the Kadec-Klee property [24] if for every sequence $\left\{x_{n}\right\}$ in $E, x_{n} \rightarrow x$ weakly and $\left\|x_{n}\right\| \rightarrow\|x\|$ it follows that $\| x_{n}-$ $x \| \rightarrow 0$.

Definition 2.2. Condition $(A)$ : The mapping $T: C \rightarrow C$ with $F(T) \neq$ $\emptyset$ is said to satisfy condition $(A)[22]$ if there is a nondecreasing function $f:[0, \infty) \rightarrow[0, \infty)$ with $f(0)=0, f(t)>0$ for all $t \in(0, \infty)$ such that $\|x-T x\| \geq f(d(x, F(T)))$ for all $x \in C$, where $d(x, F(T))=\inf \{\|x-p\|$ : $p \in F(T)\}$.

Now, we modify Condition $(A)$ for three mappings.

Definition 2.3. Condition $(B)$ : Three mappings $T_{1}, T_{2}, T_{3}: C \rightarrow C$ are said to satisfy condition $(B)$ if there is a nondecreasing function $f:[0, \infty) \rightarrow$ $[0, \infty)$ with $f(0)=0, f(t)>0$ for all $t \in(0, \infty)$ such that $a_{1}\left\|x-T_{1} x\right\|+$ $a_{2}\left\|x-T_{2} x\right\|+a_{3} T_{3} x \geq f(d(x, F))$ for all $x \in C$, where $d(x, F)=\inf \{\|x-p\|$ : $\left.p \in F=\cap_{i=1}^{3} F\left(T_{i}\right)\right\}$, where $a_{1}, a_{2}$ and $a_{3}$ are nonnegative real numbers such that $a_{1}+a_{2}+a_{3}=1$.

Note that condition $(B)$ reduces to condition $(A)$ when $T_{1}=T_{2}=T_{3}=T$ and hence is more general than the demicompactness of $T_{1}, T_{2}$ and $T_{3}$ [22]. A mapping $T: C \rightarrow C$ is called: (1) demicompact if any bounded sequence $\left\{x_{n}\right\}$ in $C$ such that $\left\{x_{n}-T x_{n}\right\}$ converges has a convergent subsequence; (2) semicompact (or hemicompact) if any bounded sequence $\left\{x_{n}\right\}$ in $C$ such that $\left\{x_{n}-T x_{n}\right\} \rightarrow 0$ as $n \rightarrow \infty$ has a convergent subsequence. Every demicompact mapping is semicompact but the converse is not true in general.

Senter and Dotson [22] have approximated fixed points of a nonexpansive mapping $T$ by Mann iterates whereas Maiti and Ghosh [10] and Tan and $\mathrm{Xu}$ [25] have approximated the fixed points using Ishikawa iterates under the condition $(A)$ of [22]. Tan and $\mathrm{Xu}$ [25] pointed out that condition $(A)$ is weaker than the compactness of $C$. We shall use condition $(B)$ instead of compactness of $C$ to study the strong convergence of $\left\{x_{n}\right\}$ defined by iteration scheme (13).

Lemma 2.1. (See [25]) Let $\left\{\alpha_{n}\right\}_{n=1}^{\infty},\left\{\beta_{n}\right\}_{n=1}^{\infty}$ and $\left\{r_{n}\right\}_{n=1}^{\infty}$ be sequences of nonnegative numbers satisfying the inequality

$$
\alpha_{n+1} \leq\left(1+\beta_{n}\right) \alpha_{n}+r_{n}, \forall n \geq 1 .
$$

If $\sum_{n=1}^{\infty} \beta_{n}<\infty$ and $\sum_{n=1}^{\infty} r_{n}<\infty$, then

(i) $\lim _{n \rightarrow \infty} \alpha_{n}$ exists;

(ii) In particular, if $\left\{\alpha_{n}\right\}_{n=1}^{\infty}$ has a subsequence which converges strongly to zero, then $\lim _{n \rightarrow \infty} \alpha_{n}=0$.

Lemma 2.2. (See [21]) Let $E$ be a uniformly convex Banach space and $0<\alpha \leq t_{n} \leq \beta<1$ for all $n \in \mathbb{N}$. Suppose further that $\left\{x_{n}\right\}$ and $\left\{y_{n}\right\}$ are 
sequences of $E$ such that $\limsup _{n \rightarrow \infty}\left\|x_{n}\right\| \leq a$, $\limsup _{n \rightarrow \infty}\left\|y_{n}\right\| \leq a$ and $\lim _{n \rightarrow \infty}\left\|t_{n} x_{n}+\left(1-t_{n}\right) y_{n}\right\|=a$ hold for some $a \geq 0$. Then $\lim _{n \rightarrow \infty}\left\|x_{n}-y_{n}\right\|$ $=0$.

Lemma 2.3. (See [24]) Let $E$ be a real reflexive Banach space with its dual $E^{*}$ has the Kadec-Klee property. Let $\left\{x_{n}\right\}$ be a bounded sequence in $E$ and $p, q \in w_{w}\left(x_{n}\right)$ (where $w_{w}\left(x_{n}\right)$ denotes the set of all weak subsequential limits of $\left.\left\{x_{n}\right\}\right)$. Suppose $\lim _{n \rightarrow \infty}\left\|t x_{n}+(1-t) p-q\right\|$ exists for all $t \in[0,1]$. Then $p=q$.

Lemma 2.4. (See [24]) Let $K$ be a nonempty convex subset of a uniformly convex Banach space $E$. Then there exists a strictly increasing continuous convex function $\phi:[0, \infty) \rightarrow[0, \infty)$ with $\phi(0)=0$ such that for each Lipschitzian mapping $T: C \rightarrow C$ with the Lipschitz constant $L$,

$$
\|t T x+(1-t) T y-T(t x+(1-t) y)\| \leq L \phi^{-1}\left(\|x-y\|-\frac{1}{L}\|T x-T y\|\right)
$$

for all $x, y \in K$ and all $t \in[0,1]$.

Proposition 2.1. Let $C$ be a nonempty subset of a Banach space $E$ and $T_{1}, T_{2}, T_{3}: C \rightarrow C$ be three generalized asymptotically quasi-nonexpansive mappings. Then there exist nonnegative real sequences $\left\{r_{n}\right\}$ and $\left\{s_{n}\right\}$ in $[0, \infty)$ with $r_{n} \rightarrow 0$ and $s_{n} \rightarrow 0$ such that

$$
\begin{aligned}
& \left\|T_{1}^{n} x-p\right\| \leq\left(1+r_{n}\right)\|x-p\|+s_{n}\left\|x-T^{n} x\right\|, \\
& \left\|T_{2}^{n} x-p\right\| \leq\left(1+r_{n}\right)\|x-p\|+s_{n}\left\|x-T^{n} x\right\|,
\end{aligned}
$$

and

$$
\left\|T_{3}^{n} x-p\right\| \leq\left(1+r_{n}\right)\|x-p\|+s_{n}\left\|x-T^{n} x\right\|,
$$

for all $x \in C, p \in F(T)$ and $n \geq 1$.

Proof. Since $T_{1}, T_{2}, T_{3}: C \rightarrow C$ are three generalized asymptotically quasinonexpansive mappings, there exist nonnegative real sequences $\left\{r_{n_{1}}\right\},\left\{r_{n_{2}}\right\}$, $\left\{r_{n_{3}}\right\},\left\{s_{n_{1}}\right\},\left\{s_{n_{2}}\right\}$ and $\left\{s_{n_{3}}\right\}$ in $[0, \infty)$ with $r_{n_{i}} \rightarrow 0$ and $s_{n_{i}} \rightarrow 0$ as $n \rightarrow \infty$ for all $i=1,2,3$ such that

$$
\begin{aligned}
& \left\|T_{1}^{n} x-p\right\| \leq\left(1+r_{n_{1}}\right)\|x-p\|+s_{n_{1}}\left\|x-T^{n} x\right\|, \\
& \left\|T_{2}^{n} x-p\right\| \leq\left(1+r_{n_{2}}\right)\|x-p\|+s_{n_{2}}\left\|x-T^{n} x\right\|,
\end{aligned}
$$

and

$$
\left\|T_{3}^{n} x-p\right\| \leq\left(1+r_{n_{3}}\right)\|x-p\|+s_{n_{3}}\left\|x-T^{n} x\right\|,
$$

for all $x \in C, p \in F(T)$ and $n \geq 1$.

Setting

$$
r_{n}=\max \left\{r_{n_{i}}: i=1,2,3\right\}, \quad s_{n}=\max \left\{s_{n_{i}}: i=1,2,3\right\}
$$


then we get that there exist nonnegative real sequences $\left\{r_{n}\right\}$ and $\left\{s_{n}\right\}$ with $r_{n} \rightarrow 0$ and $s_{n} \rightarrow 0$ as $n \rightarrow \infty$ such that

$$
\begin{aligned}
\left\|T_{1}^{n} x-p\right\| & \leq\left(1+r_{n_{1}}\right)\|x-p\|+s_{n_{1}}\left\|x-T^{n} x\right\| \\
& \leq\left(1+r_{n}\right)\|x-p\|+s_{n}\left\|x-T^{n} x\right\|, \\
\left\|T_{2}^{n} x-p\right\| & \leq\left(1+r_{n_{2}}\right)\|x-p\|+s_{n_{2}}\left\|x-T^{n} x\right\| \\
& \leq\left(1+r_{n}\right)\|x-p\|+s_{n}\left\|x-T^{n} x\right\|,
\end{aligned}
$$

and

$$
\begin{aligned}
\left\|T_{3}^{n} x-p\right\| & \leq\left(1+r_{n_{3}}\right)\|x-p\|+s_{n_{3}}\left\|x-T^{n} x\right\| \\
& \leq\left(1+r_{n}\right)\|x-p\|+s_{n}\left\|x-T^{n} x\right\|,
\end{aligned}
$$

for all $x \in C, p \in F(T)$ and $n \geq 1$.

\section{Strong Convergence Theorems}

In this section, we prove some strong convergence theorems of iteration scheme (12) for three generalized asymptotically quasi-nonexpansive mappings in a Banach space. First, we shall need the following lemmas.

Lemma 3.1. Let $E$ be a Banach space and let $C$ be a nonempty closed convex subset of $E$. Let $T_{1}, T_{2}, T_{3}: C \rightarrow C$ be three generalized asymptotically quasi-nonexpansive mappings with sequences $\left\{r_{n}\right\}$ and $\left\{s_{n}\right\}$ as defined in proposition 2.1 such that $\sum_{n=1}^{\infty} \frac{r_{n}+2 s_{n}}{1-s_{n}}<\infty$ and $F=\cap_{i=1}^{3} F\left(T_{i}\right) \neq \emptyset$. Let $\left\{x_{n}\right\}$ be the iteration scheme defined by (12). Then $\lim _{n \rightarrow \infty}\left\|x_{n}-q\right\|$ and $\lim _{n \rightarrow \infty} d\left(x_{n}, F\right)$ both exist for all $q \in F$.

Proof. Let $q \in F$, then from (12), we have

$$
\begin{aligned}
\left\|z_{n}-q\right\| \leq & \left\|\left(1-\gamma_{n}\right) x_{n}+\gamma_{n} T_{3}^{n} x_{n}-q\right\| \\
= & \left\|\left(1-\gamma_{n}\right)\left(x_{n}-q\right)+\gamma_{n}\left(T_{3}^{n} x_{n}-q\right)\right\| \\
\leq & \left(1-\gamma_{n}\right)\left\|x_{n}-q\right\|+\gamma_{n}\left\|T_{3}^{n} x_{n}-q\right\| \\
\leq & \left(1-\gamma_{n}\right)\left\|x_{n}-q\right\|+\gamma_{n}\left[\left(1+r_{n}\right)\left\|x_{n}-q\right\|\right. \\
& \left.+s_{n}\left\|x_{n}-T_{3}^{n} x_{n}\right\|\right] \\
\leq & \left(1+r_{n}\right)\left[\left(1-\gamma_{n}\right)\left\|x_{n}-q\right\|+\gamma_{n}\left\|x_{n}-q\right\|\right] \\
& +s_{n}\left\|x_{n}-T_{3}^{n} x_{n}\right\| \\
\leq & \left(1+r_{n}\right)\left\|x_{n}-q\right\|+s_{n}\left\|x_{n}-T_{3}^{n} x_{n}\right\| .
\end{aligned}
$$

Now, we have

$$
\begin{aligned}
\left\|x_{n}-T_{3}^{n} x_{n}\right\| & \leq\left\|x_{n}-q\right\|+\left\|T_{3}^{n} x_{n}-q\right\| \\
& \leq\left\|x_{n}-q\right\|+\left(1+r_{n}\right)\left\|x_{n}-q\right\|+s_{n}\left\|x_{n}-T_{3}^{n} x_{n}\right\| \\
& =\left(2+r_{n}\right)\left\|x_{n}-q\right\|+s_{n}\left\|x_{n}-T_{3}^{n} x_{n}\right\|
\end{aligned}
$$


which implies that

$$
\left\|x_{n}-T_{3}^{n} x_{n}\right\| \leq\left(\frac{2+r_{n}}{1-s_{n}}\right)\left\|x_{n}-q\right\| .
$$

Now using (20) in (19), we have

$$
\begin{aligned}
\left\|z_{n}-q\right\| & \leq\left(1+r_{n}\right)\left\|x_{n}-q\right\|+s_{n}\left(\frac{2+r_{n}}{1-s_{n}}\right)\left\|x_{n}-q\right\| \\
& =\left[\left(1+r_{n}\right)+s_{n}\left(\frac{2+r_{n}}{1-s_{n}}\right)\right]\left\|x_{n}-q\right\| \\
& =\left(\frac{1+r_{n}+s_{n}}{1-s_{n}}\right)\left\|x_{n}-q\right\| .
\end{aligned}
$$

Again from (12), we have

$$
\begin{aligned}
\left\|y_{n}-q\right\| \leq & \left\|\left(1-\beta_{n}\right) z_{n}+\beta_{n} T_{2}^{n} z_{n}-q\right\| \\
= & \left\|\left(1-\beta_{n}\right)\left(z_{n}-q\right)+\beta_{n}\left(T_{2}^{n} z_{n}-q\right)\right\| \\
\leq & \left(1-\beta_{n}\right)\left\|z_{n}-q\right\|+\beta_{n}\left\|T_{2}^{n} z_{n}-q\right\| \\
\leq & \left(1-\beta_{n}\right)\left\|z_{n}-q\right\|+\beta_{n}\left[\left(1+r_{n}\right)\left\|z_{n}-q\right\|\right. \\
& \left.+s_{n}\left\|z_{n}-T_{2}^{n} z_{n}\right\|\right] \\
\leq & \left(1+r_{n}\right)\left[\left(1-\beta_{n}\right)\left\|z_{n}-q\right\|+\beta_{n}\left\|z_{n}-q\right\|\right] \\
& +s_{n}\left\|z_{n}-T_{2}^{n} z_{n}\right\| \\
\leq & \left(1+r_{n}\right)\left\|z_{n}-q\right\|+s_{n}\left\|z_{n}-T_{2}^{n} z_{n}\right\| .
\end{aligned}
$$

Now, we have

$$
\begin{aligned}
\left\|z_{n}-T_{2}^{n} z_{n}\right\| & \leq\left\|z_{n}-q\right\|+\left\|T_{2}^{n} z_{n}-q\right\| \\
& \leq\left\|z_{n}-q\right\|+\left(1+r_{n}\right)\left\|z_{n}-q\right\|+s_{n}\left\|z_{n}-T_{2}^{n} z_{n}\right\| \\
& =\left(2+r_{n}\right)\left\|z_{n}-q\right\|+s_{n}\left\|z_{n}-T_{2}^{n} z_{n}\right\|
\end{aligned}
$$

which implies that

$$
\left\|z_{n}-T_{2}^{n} z_{n}\right\| \leq\left(\frac{2+r_{n}}{1-s_{n}}\right)\left\|z_{n}-q\right\| .
$$

Using (23) in (22), we have

$$
\begin{aligned}
\left\|y_{n}-q\right\| & \leq\left(1+r_{n}\right)\left\|z_{n}-q\right\|+s_{n}\left(\frac{2+r_{n}}{1-s_{n}}\right)\left\|z_{n}-q\right\| \\
& =\left[\left(1+r_{n}\right)+s_{n}\left(\frac{2+r_{n}}{1-s_{n}}\right)\right]\left\|z_{n}-q\right\| \\
& =\left(\frac{1+r_{n}+s_{n}}{1-s_{n}}\right)\left\|z_{n}-q\right\| .
\end{aligned}
$$

Now substituting (21) in (24), we get

$$
\left\|y_{n}-q\right\| \leq\left(\frac{1+r_{n}+s_{n}}{1-s_{n}}\right)\left(\frac{1+r_{n}+s_{n}}{1-s_{n}}\right)\left\|x_{n}-q\right\|
$$




$$
=\left(\frac{1+r_{n}+s_{n}}{1-s_{n}}\right)^{2}\left\|x_{n}-q\right\| .
$$

Finally, from (13), we have

$$
\begin{aligned}
\left\|x_{n+1}-q\right\| \leq & \left\|\left(1-\alpha_{n}\right) y_{n}+\alpha_{n} T_{1}^{n} y_{n}-q\right\| \\
= & \left\|\left(1-\alpha_{n}\right)\left(y_{n}-q\right)+\alpha_{n}\left(T_{1}^{n} y_{n}-q\right)\right\| \\
\leq & \left(1-\alpha_{n}\right)\left\|y_{n}-q\right\|+\alpha_{n}\left\|T_{1}^{n} y_{n}-q\right\| \\
\leq & \left(1-\alpha_{n}\right)\left\|y_{n}-q\right\|+\alpha_{n}\left[\left(1+r_{n}\right)\left\|y_{n}-q\right\|\right. \\
& \left.+s_{n}\left\|y_{n}-T_{1}^{n} y_{n}\right\|\right] \\
\leq & \left(1+r_{n}\right)\left[\left(1-\alpha_{n}\right)\left\|y_{n}-q\right\|+\alpha_{n}\left\|y_{n}-q\right\|\right] \\
& +s_{n}\left\|y_{n}-T_{1}^{n} y_{n}\right\| \\
\leq & \left(1+r_{n}\right)\left\|y_{n}-q\right\|+s_{n}\left\|y_{n}-T_{1}^{n} y_{n}\right\| .
\end{aligned}
$$

Now, we have

$$
\begin{aligned}
\left\|y_{n}-T_{1}^{n} y_{n}\right\| & \leq\left\|y_{n}-q\right\|+\left\|T_{1}^{n} y_{n}-q\right\| \\
& \leq\left\|y_{n}-q\right\|+\left(1+r_{n}\right)\left\|y_{n}-q\right\|+s_{n}\left\|y_{n}-T_{1}^{n} y_{n}\right\| \\
& =\left(2+r_{n}\right)\left\|y_{n}-q\right\|+s_{n}\left\|y_{n}-T_{1}^{n} y_{n}\right\|
\end{aligned}
$$

which implies that

$$
\left\|y_{n}-T_{1}^{n} y_{n}\right\| \leq\left(\frac{2+r_{n}}{1-s_{n}}\right)\left\|y_{n}-q\right\| .
$$

Substituting (27) in (26), we obtain

$$
\begin{aligned}
\left\|x_{n+1}-q\right\| & \leq\left(1+r_{n}\right)\left\|y_{n}-q\right\|+s_{n}\left(\frac{2+r_{n}}{1-s_{n}}\right)\left\|y_{n}-q\right\| \\
& =\left[\left(1+r_{n}\right)+s_{n}\left(\frac{2+r_{n}}{1-s_{n}}\right)\right]\left\|y_{n}-q\right\| \\
& =\left(\frac{1+r_{n}+s_{n}}{1-s_{n}}\right)\left\|y_{n}-q\right\| .
\end{aligned}
$$

Now substituting (25) in (28), we get

$$
\begin{aligned}
\left\|x_{n+1}-q\right\| & \leq\left(\frac{1+r_{n}+s_{n}}{1-s_{n}}\right)\left(\frac{1+r_{n}+s_{n}}{1-s_{n}}\right)^{2}\left\|x_{n}-q\right\| \\
& =\left(\frac{1+r_{n}+s_{n}}{1-s_{n}}\right)^{3}\left\|x_{n}-q\right\| \\
& =\left[1+\left(\frac{r_{n}+2 s_{n}}{1-s_{n}}\right)\right]^{3}\left\|x_{n}-q\right\| \\
& =\left(1+h_{n}\right)^{3}\left\|x_{n}-q\right\| \\
& \leq\left(1+Q h_{n}\right)\left\|x_{n}-q\right\|
\end{aligned}
$$

where $h_{n}=\frac{r_{n}+2 s_{n}}{1-s_{n}}$ and for some $Q>0$. Since by hypothesis $\sum_{n=1}^{\infty} \frac{r_{n}+2 s_{n}}{1-s_{n}}<$ $\infty$, it follows that $\sum_{n=1}^{\infty} h_{n}<\infty$. 
For any $q \in F$, from (29), we obtain the following inequality

$$
d\left(x_{n+1}, F\right) \leq\left(1+Q h_{n}\right) d\left(x_{n}, F\right) .
$$

Applying Lemma 2.1 in (29) and (30), we have $\lim _{n \rightarrow \infty}\left\|x_{n}-q\right\|$ and $d\left(x_{n}, F\right)$ both exist. This completes the proof.

Lemma 3.2. Let $E$ be a uniformly convex Banach space and let $C$ be a nonempty closed convex subset of $E$. Let $T_{1}, T_{2}, T_{3}: C \rightarrow C$ be three uniformly L-Lipschitzian and generalized asymptotically quasi-nonexpansive mappings with sequences $\left\{r_{n}\right\}$ and $\left\{s_{n}\right\}$ as defined in proposition 2.1 such that $\sum_{n=1}^{\infty} \frac{r_{n}+2 s_{n}}{1-s_{n}}<\infty$ and $F=\cap_{i=1}^{3} F\left(T_{i}\right) \neq \emptyset$. Let $\left\{x_{n}\right\}$ be the iteration scheme defined by (12), where $\left\{\alpha_{n}\right\},\left\{\beta_{n}\right\}$ and $\left\{\gamma_{n}\right\}$ are sequences in $[\rho, 1-\rho]$ for all $n \in \mathbb{N}$ and for some $\rho \in(0,1)$. Then $\lim _{n \rightarrow \infty}\left\|x_{n}-T_{i} x_{n}\right\|=0$ for all $i=1,2,3$.

Proof. By Lemma 3.1, $\lim _{n \rightarrow \infty}\left\|x_{n}-q\right\|$ exists for all $q \in F$, so we can assume that $\lim _{n \rightarrow \infty}\left\|x_{n}-q\right\|=c$. Then $c>0$ otherwise there is nothing to prove.

Now (21) and (25) implies that

$$
\limsup _{n \rightarrow \infty}\left\|z_{n}-p\right\| \leq c
$$

and

$$
\limsup _{n \rightarrow \infty}\left\|y_{n}-p\right\| \leq c
$$

Also

$$
\left\|T_{1}^{n} y_{n}-p\right\| \leq\left(1+r_{n}\right)\left\|y_{n}-p\right\|+s_{n}\left\|y_{n}-T_{1}^{n} y_{n}\right\|
$$

and so

$$
\limsup _{n \rightarrow \infty}\left\|T_{1}^{n} y_{n}-p\right\| \leq c
$$

Since

$$
c=\left\|x_{n+1}-p\right\|=\left\|\left(1-\alpha_{n}\right)\left(y_{n}-p\right)+\alpha_{n}\left(T_{1}^{n} y_{n}-p\right)\right\| .
$$

It follows from Lemma 2.2 that

$$
\lim _{n \rightarrow \infty}\left\|T_{1}^{n} y_{n}-y_{n}\right\|=0 .
$$

Again note that

$$
\begin{aligned}
&\left\|T_{3}^{n} x_{n}-p\right\| \leq\left(1+r_{n}\right)\left\|x_{n}-p\right\|+s_{n}\left\|x_{n}-T_{3}^{n} x_{n}\right\| \\
&\left\|T_{2}^{n} z_{n}-p\right\| \leq\left(1+r_{n}\right)\left\|z_{n}-p\right\|+s_{n}\left\|z_{n}-T_{2}^{n} z_{n}\right\| .
\end{aligned}
$$

Hence, from above inequalities, we obtain

$$
\limsup _{n \rightarrow \infty}\left\|T_{3}^{n} x_{n}-p\right\| \leq c
$$


and

$$
\limsup _{n \rightarrow \infty}\left\|T_{2}^{n} z_{n}-p\right\| \leq c .
$$

Further, note that

$$
\begin{aligned}
\left\|y_{n}-p\right\| & \leq\left\|y_{n}-T_{1}^{n} y_{n}\right\|+\left\|T_{1}^{n} y_{n}-p\right\| \\
& \leq\left\|y_{n}-T_{1}^{n} y_{n}\right\|+\left(1+r_{n}\right)\left\|y_{n}-p\right\|+s_{n}\left\|y_{n}-T_{1}^{n} y_{n}\right\| .
\end{aligned}
$$

It follows from (32) and (34) that

$$
c \leq \liminf _{n \rightarrow \infty}\left\|y_{n}-p\right\| .
$$

From (32) and (37), we get

$$
\lim _{n \rightarrow \infty}\left\|y_{n}-p\right\|=c .
$$

Now, we have

$$
c=\lim _{n \rightarrow \infty}\left\|y_{n}-p\right\|=\left\|\left(1-\beta_{n}\right)\left(z_{n}-p\right)+\beta_{n}\left(T_{2}^{n} z_{n}-p\right)\right\| .
$$

It follows from (31), (36) and Lemma 2.2 that

$$
\lim _{n \rightarrow \infty}\left\|T_{2}^{n} z_{n}-z_{n}\right\|=0 \text {. }
$$

Again note that

$$
\begin{aligned}
\left\|z_{n}-p\right\| & \leq\left\|z_{n}-T_{2}^{n} z_{n}\right\|+\left\|T_{2}^{n} z_{n}-p\right\| \\
& \leq\left\|z_{n}-T_{2}^{n} z_{n}\right\|+\left(1+r_{n}\right)\left\|z_{n}-p\right\|+s_{n}\left\|z_{n}-T_{2}^{n} z_{n}\right\| .
\end{aligned}
$$

It follows from (31) and (40) that

$$
c \leq \liminf _{n \rightarrow \infty}\left\|z_{n}-p\right\| .
$$

From (31) and (41), we get

$$
\lim _{n \rightarrow \infty}\left\|z_{n}-p\right\|=c .
$$

Now, we see that

$$
c=\lim _{n \rightarrow \infty}\left\|z_{n}-p\right\|=\left\|\left(1-\gamma_{n}\right)\left(x_{n}-p\right)+\gamma_{n}\left(T_{3}^{n} x_{n}-p\right)\right\| .
$$

It follows from Lemma 2.2 that

$$
\lim _{n \rightarrow \infty}\left\|T_{3}^{n} x_{n}-x_{n}\right\|=0 .
$$

Again note that

$$
\begin{aligned}
\left\|x_{n}-z_{n}\right\| & =\gamma_{n}\left\|x_{n}-T_{3}^{n} x_{n}\right\| \\
& \leq(1-\rho)\left\|x_{n}-T_{3}^{n} x_{n}\right\| .
\end{aligned}
$$

Using (43) in (45), we get

$$
\lim _{n \rightarrow \infty}\left\|x_{n}-z_{n}\right\|=0 .
$$

Further, note that

$$
\left\|x_{n}-y_{n}\right\|=\beta_{n}\left\|z_{n}-T_{2}^{n} z_{n}\right\|
$$




$$
\leq(1-\rho)\left\|z_{n}-T_{2}^{n} z_{n}\right\|
$$

Using (40) in (47), we get

$$
\lim _{n \rightarrow \infty}\left\|x_{n}-y_{n}\right\|=0 .
$$

Note that

$$
\left\|x_{n}-T_{2}^{n} z_{n}\right\| \leq\left\|x_{n}-z_{n}\right\|+\left\|z_{n}-T_{2}^{n} z_{n}\right\| .
$$

Using (40) and (46) in (49), we get

$$
\lim _{n \rightarrow \infty}\left\|x_{n}-T_{2}^{n} z_{n}\right\|=0 .
$$

Since $T_{2}$ is uniformly $L$-Lipschitzian, we have

$$
\begin{aligned}
\left\|x_{n}-T_{2}^{n} x_{n}\right\| & \leq\left\|x_{n}-T_{2}^{n} z_{n}\right\|+\left\|T_{2}^{n} z_{n}-T_{2}^{n} x_{n}\right\| \\
& \leq\left\|x_{n}-T_{2}^{n} z_{n}\right\|+L\left\|z_{n}-x_{n}\right\| .
\end{aligned}
$$

Using (46) and (50) in (51), we get

$$
\lim _{n \rightarrow \infty}\left\|x_{n}-T_{2}^{n} x_{n}\right\|=0 .
$$

Again notice that

$$
\left\|x_{n}-T_{1}^{n} y_{n}\right\| \leq\left\|x_{n}-y_{n}\right\|+\left\|y_{n}-T_{1}^{n} y_{n}\right\| .
$$

Using (34) and (48) in (53), we get

$$
\lim _{n \rightarrow \infty}\left\|x_{n}-T_{1}^{n} y_{n}\right\|=0 .
$$

Since $T_{1}$ is uniformly $L$-Lipschitzian, we obtain

$$
\begin{aligned}
\left\|x_{n}-T_{1}^{n} x_{n}\right\| & \leq\left\|x_{n}-T_{1}^{n} y_{n}\right\|+\left\|T_{1}^{n} x_{n}-T_{1}^{n} y_{n}\right\| \\
& \leq\left\|x_{n}-T_{1}^{n} y_{n}\right\|+L\left\|x_{n}-y_{n}\right\| .
\end{aligned}
$$

Using (48) and (54) in (55), we get

$$
\lim _{n \rightarrow \infty}\left\|x_{n}-T_{1}^{n} x_{n}\right\|=0 .
$$

By the definitions of $x_{n+1}$, we have

$$
\left\|x_{n}-x_{n+1}\right\| \leq\left\|x_{n}-y_{n}\right\|+\left\|T_{1}^{n} y_{n}-y_{n}\right\| .
$$

Using (34) and (48) in (57), we get

$$
\lim _{n \rightarrow \infty}\left\|x_{n}-x_{n+1}\right\|=0 .
$$

By (56), (57) and since $T_{1}$ is uniformly $L$-Lipschitzian, we have

$$
\begin{aligned}
\left\|x_{n}-T_{1} x_{n}\right\| \leq & \left\|x_{n}-x_{n+1}\right\|+\left\|x_{n+1}-T_{1}^{n+1} x_{n+1}\right\| \\
& +\left\|T_{1}^{n+1} x_{n+1}-T_{1}^{n+1} x_{n}\right\|+\left\|T_{1}^{n+1} x_{n}-T_{1} x_{n}\right\| \\
\leq & \left\|x_{n}-x_{n+1}\right\|+\left\|x_{n+1}-T_{1}^{n+1} x_{n+1}\right\| \\
& +L\left\|x_{n+1}-x_{n}\right\|+L\left\|T_{1}^{n} x_{n}-x_{n}\right\| \\
= & (1+L)\left\|x_{n}-x_{n+1}\right\|+\left\|x_{n+1}-T_{1}^{n+1} x_{n+1}\right\| \\
& +L\left\|T_{1}^{n} x_{n}-x_{n}\right\| \rightarrow 0 \text { as } n \rightarrow \infty
\end{aligned}
$$


Similarly, we can prove that

$$
\left\|x_{n}-T_{2} x_{n}\right\|=0 \text { and }\left\|x_{n}-T_{3} x_{n}\right\|=0 .
$$

This completes the proof.

Theorem 3.1. Let $E$ be a real Banach space and $C$ be a nonempty closed convex subset of $E$. Let $T_{1}, T_{2}, T_{3}: C \rightarrow C$ be three generalized asymptotically quasi-nonexpansive mappings with sequences $\left\{r_{n}\right\}$ and $\left\{s_{n}\right\}$ as defined in proposition 2.1 such that $\sum_{n=1}^{\infty} \frac{r_{n}+2 s_{n}}{1-s_{n}}<\infty$ and $F=\cap_{i=1}^{3} F\left(T_{i}\right)$ is closed. Let $\left\{x_{n}\right\}$ be the iteration scheme defined by (12), where $\left\{\alpha_{n}\right\},\left\{\beta_{n}\right\}$ and $\left\{\gamma_{n}\right\}$ are sequences in $[\rho, 1-\rho]$ for all $n \in \mathbb{N}$ and for some $\rho \in(0,1)$. Then $\left\{x_{n}\right\}$ converges strongly to a common fixed point of the mappings $T_{1}, T_{2}$ and $T_{3}$ if and only if $\liminf _{n \rightarrow \infty} d\left(x_{n}, F\right)=0$, where $d(x, F)=\inf \{\|x-p\|: p \in F\}$

Proof. Necessity is obvious. Conversely, suppose that $\liminf _{n \rightarrow \infty} d\left(x_{n}, F\right)=$ 0 . As proved in Lemma 3.1, for all $q \in F, \lim _{n \rightarrow \infty} d\left(x_{n}, F\right)$ exists. Thus by the hypothesis $\lim _{n \rightarrow \infty} d\left(x_{n}, F\right)=0$.

Now, we show that $\left\{x_{n}\right\}$ is a Cauchy sequence in $C$. From (29), we know that

$$
\begin{aligned}
\left\|x_{n+1}-q\right\| & \leq\left(1+Q h_{n}\right)\left\|x_{n}-q\right\| \\
& =\left\|x_{n}-q\right\|+Q^{\prime} h_{n},
\end{aligned}
$$

for some $Q^{\prime}>0$ with $\sum_{n=1}^{\infty} h_{n}<\infty$.

Now, given $\varepsilon>0$, since $\lim _{n \rightarrow \infty} d\left(x_{n}, F\right)=0$ and $\sum_{n=1}^{\infty} h_{n}<\infty$, there exists a natural number $n_{1}>0$ such that for all $n \geq n_{1}, d\left(x_{n}, F\right)<\frac{\varepsilon}{5}$ and $\sum_{j=1}^{\infty} h_{j}<\frac{\varepsilon}{4 Q^{\prime}}$. So, we get $d\left(x_{n_{1}}, F\right)<\frac{\varepsilon}{4}$ and $\sum_{j=n_{1}}^{\infty} h_{j}<\frac{\varepsilon}{4 Q^{\prime}}$. This means that there exists a $q_{1} \in F$ such that $\left\|x_{n_{1}}-q_{1}\right\| \leq \frac{\varepsilon}{4}$. Hence, for all integers $n \geq n_{1}$ and $m \geq 1$, we obtain from (61) that

$$
\begin{aligned}
\left\|x_{n+m}-x_{n}\right\| & \leq\left\|x_{n+m}-q_{1}\right\|+\left\|x_{n}-q_{1}\right\| \\
& \leq\left\|x_{n_{1}}-q_{1}\right\|+Q^{\prime} \sum_{j=n_{1}}^{n+m-1} h_{j}+\left\|x_{n_{1}}-q_{1}\right\|+Q^{\prime} \sum_{j=n_{1}}^{n+m-1} h_{j} \\
& \leq\left\|x_{n_{1}}-q_{1}\right\|+Q^{\prime} \sum_{j=n_{1}}^{\infty} h_{j}+\left\|x_{n_{1}}-q_{1}\right\|+Q^{\prime} \sum_{j=n_{1}}^{\infty} h_{j} \\
& =2\left(\left\|x_{n_{1}}-q_{1}\right\|+Q^{\prime} \sum_{j=n_{1}}^{\infty} h_{j}\right) \\
& <2\left(\frac{\varepsilon}{4}+Q^{\prime} \cdot \frac{\varepsilon}{4 Q^{\prime}}\right)=\varepsilon .
\end{aligned}
$$

This proves that $\left\{x_{n}\right\}$ is a Cauchy sequence in $C$. Thus, the completeness of $E$ implies that $\left\{x_{n}\right\}$ must be convergent. Assume that $\lim _{n \rightarrow \infty} x_{n}=p^{*}$. We will prove that $p^{*}$ is a common fixed point of $T_{1}, T_{2}$ and $T_{3}$, that is, we will 
show that $p^{*} \in F=\cap_{i=1}^{3} F\left(T_{i}\right)$. Since $C$ is closed, therefore $p^{*} \in C$. Next, we show that $p^{*} \in F$. Now $\lim _{n \rightarrow \infty} d\left(x_{n}, F\right)=0$ gives that $d\left(p^{*}, F\right)=0$. Since $F$ is closed, $p^{*} \in F$. Thus, $p^{*}$ is a common fixed point of the mappings $T_{1}, T_{2}$ and $T_{3}$. This completes the proof.

We deduce the following result as corollary from Theorem 3.1 as follows.

Corollary 3.1. Let $E$ be a real Banach space and $C$ be a nonempty closed convex subset of $E$. Let $T_{1}, T_{2}, T_{3}: C \rightarrow C$ be three generalized asymptotically quasi-nonexpansive mappings with sequences $\left\{r_{n}\right\}$ and $\left\{s_{n}\right\}$ as defined in proposition 2.1 such that $\sum_{n=1}^{\infty} \frac{r_{n}+2 s_{n}}{1-s_{n}}<\infty$ and $F=\cap_{i=1}^{3} F\left(T_{i}\right)$ is closed. Let $\left\{x_{n}\right\}$ be the iteration scheme defined by (12), where $\left\{\alpha_{n}\right\},\left\{\beta_{n}\right\}$ and $\left\{\gamma_{n}\right\}$ are sequences in $[\rho, 1-\rho]$ for all $n \in \mathbb{N}$ and for some $\rho \in(0,1)$. Then $\left\{x_{n}\right\}$ converges strongly to a point $p \in F$ if and only if there exists some subsequence $\left\{x_{n_{j}}\right\}$ of $\left\{x_{n}\right\}$ which converges to a point $p \in F$.

Theorem 3.2. Let $E$ be a real Banach space and $C$ be a nonempty closed convex subset of $E$. Let $T_{1}, T_{2}, T_{3}: C \rightarrow C$ be three generalized asymptotically quasi-nonexpansive mappings with sequences $\left\{r_{n}\right\}$ and $\left\{s_{n}\right\}$ as defined in proposition 2.1 such that $\sum_{n=1}^{\infty} \frac{r_{n}+2 s_{n}}{1-s_{n}}<\infty$ and $F=\cap_{i=1}^{3} F\left(T_{i}\right) \neq \emptyset$. Let $\left\{x_{n}\right\}$ be the iteration scheme defined by (12), where $\left\{\alpha_{n}\right\},\left\{\beta_{n}\right\}$ and $\left\{\gamma_{n}\right\}$ are sequences in $[\rho, 1-\rho]$ for all $n \in \mathbb{N}$ and for some $\rho \in(0,1)$. Then $\liminf \operatorname{in}_{n \rightarrow \infty} d\left(x_{n}, F\right)=\limsup _{n \rightarrow \infty} d\left(x_{n}, F\right)=0$ if $\left\{x_{n}\right\}$ converges to a unique point in $F$.

Proof. Let $p \in F$. Since $\left\{x_{n}\right\}$ converges to $p, \lim _{n \rightarrow \infty} d\left(x_{n}, p\right)=0$. So, for a given $\varepsilon>0$, there exists $n_{1} \in \mathbb{N}$ such that

$$
d\left(x_{n}, p\right)<\varepsilon \text { for all } n \geq n_{1} .
$$

Taking the infimum over $p \in F$, we obtain that

$$
d\left(x_{n}, F\right)<\varepsilon \text { for all } n \geq n_{1} .
$$

This means that $\lim _{n \rightarrow \infty} d\left(x_{n}, F\right)=0$. Thus we obtain that

$$
\liminf _{n \rightarrow \infty} d\left(x_{n}, F\right)=\limsup _{n \rightarrow \infty} d\left(x_{n}, F\right)=0 .
$$

This completes the proof.

As an application of Theorem 3.1, we establish some strong convergence results as follows.

Theorem 3.3. Let $E$ be a real Banach space and $C$ be a nonempty closed convex subset of $E$. Let $T_{1}, T_{2}, T_{3}: C \rightarrow C$ be three uniformly L-Lipschitzian and generalized asymptotically quasi-nonexpansive mappings with sequences $\left\{r_{n}\right\}$ and $\left\{s_{n}\right\}$ as defined in proposition 2.1 such that $\sum_{n=1}^{\infty} \frac{r_{n}+2 s_{n}}{1-s_{n}}<\infty$ and $F=\cap_{i=1}^{3} F\left(T_{i}\right) \neq \emptyset$. Let $\left\{x_{n}\right\}$ be the iteration scheme defined by (12), where $\left\{\alpha_{n}\right\},\left\{\beta_{n}\right\}$ and $\left\{\gamma_{n}\right\}$ are sequences in $[\rho, 1-\rho]$ for all $n \in \mathbb{N}$ and for some $\rho \in(0,1)$. If one of the mappings in $\left\{T_{i}: i=1,2,3\right\}$ is demicompact, then 
$\left\{x_{n}\right\}$ converges strongly to a common fixed point of the mappings $T_{1}, T_{2}$ and $T_{3}$.

Proof. Without loss of generality, we can assume that $T_{1}$ is demicompact. It follows from equation (59) in Lemma 3.2 that $\lim _{n \rightarrow \infty}\left\|x_{n}-T_{1} x_{n}\right\|=0$ and $\left\{x_{n}\right\}$ is bounded, by demicompactness of $T_{1}$, there exists a subsequence $\left\{x_{n_{k}}\right\}$ of $\left\{x_{n}\right\}$ that converges strongly to some $q \in C$ as $k \rightarrow \infty$. From equation (59) in Lemma 3.2 we have

$$
\lim _{k \rightarrow \infty}\left\|x_{n_{k}}-T_{1} x_{n_{k}}\right\|=\left\|q-T_{1} q\right\|=0 .
$$

This implies that $q \in F\left(T_{1}\right)$. Similarly, we can prove that $q \in F\left(T_{2}\right)$ and $q \in F\left(T_{3}\right)$. Thus, we obtain that $q \in F=\cap_{i=1}^{3} F\left(T_{i}\right)$. It follows from Lemma 3.1 and Theorem 3.1 that $\left\{x_{n}\right\}$ must converges strongly to a common fixed point of the mappings $T_{1}, T_{2}$ and $T_{3}$. This completes the proof.

Theorem 3.4. Let $E$ be a real Banach space and $C$ be a nonempty closed convex subset of $E$. Let $T_{1}, T_{2}, T_{3}: C \rightarrow C$ be three uniformly L-Lipschitzian and generalized asymptotically quasi-nonexpansive mappings with sequences $\left\{r_{n}\right\}$ and $\left\{s_{n}\right\}$ as defined in proposition 2.1 such that $\sum_{n=1}^{\infty} \frac{r_{n}+2 s_{n}}{1-s_{n}}<\infty$ and $F=\cap_{i=1}^{3} F\left(T_{i}\right) \neq \emptyset$. Let $\left\{x_{n}\right\}$ be the iteration scheme defined by (12), where $\left\{\alpha_{n}\right\},\left\{\beta_{n}\right\}$ and $\left\{\gamma_{n}\right\}$ are sequences in $[\rho, 1-\rho]$ for all $n \in \mathbb{N}$ and for some $\rho \in(0,1)$. If $T_{1}, T_{2}$ and $T_{3}$ satisfy condition $(B)$, then $\left\{x_{n}\right\}$ converges strongly to a common fixed point of the mappings $T_{1}, T_{2}$ and $T_{3}$.

Proof. By Lemma 3.2, we know that

$$
\lim _{n \rightarrow \infty}\left\|x_{n}-T_{i} x_{n}\right\|=0, \text { for } i=1,2,3 .
$$

From condition $(B)$ and (62), we get

$$
f\left(d\left(x_{n}, F\right) \leq a_{1} \cdot\left\|x_{n}-T_{1} x_{n}\right\|+a_{2} \cdot\left\|x_{n}-T_{2} x_{n}\right\|+a_{3} \cdot\left\|x_{n}-T_{3} x_{n}\right\|=0,\right.
$$

that is, $f\left(d\left(x_{n}, F\right)=0\right.$. Since $f:[0, \infty) \rightarrow[0, \infty)$ is a nondecreasing function satisfying $f(0)=0, f(t)>0$ for all $t \in(0, \infty)$, therefore we obtain

$$
\lim _{n \rightarrow \infty} d\left(x_{n}, F\right)=0 .
$$

Therefore, Theorem 3.1 implies that $\left\{x_{n}\right\}$ converges strongly to a common fixed point of the mappings $T_{1}, T_{2}$ and $T_{3}$. This completes the proof.

\section{Weak Convergence Theorems}

In this section, we prove some weak convergence theorems of iteration scheme (12) for three generalized asymptotically quasi-nonexpansive mappings in a uniformly convex Banach space such that either it satisfies the Opial property or its dual space has the Kadec-Klee property (KK-property). 
Theorem 4.1. Let $E$ be a uniformly convex Banach space satisfying Opial's condition and $C$ be a nonempty closed convex subset of $E$. Let $T_{1}, T_{2}, T_{3}: C \rightarrow$ $C$ be three uniformly L-Lipschitzian and generalized asymptotically quasinonexpansive mappings with sequences $\left\{r_{n}\right\}$ and $\left\{s_{n}\right\}$ as defined in proposition 2.1 such that $\sum_{n=1}^{\infty} \frac{r_{n}+2 s_{n}}{1-s_{n}}<\infty$ and $F=\cap_{i=1}^{3} F\left(T_{i}\right) \neq \emptyset$. Let $\left\{x_{n}\right\}$ be the iteration scheme defined by (12), where $\left\{\alpha_{n}\right\},\left\{\beta_{n}\right\}$ and $\left\{\gamma_{n}\right\}$ are sequences in $[\rho, 1-\rho]$ for all $n \in \mathbb{N}$ and for some $\rho \in(0,1)$. If the mappings $I-T_{i}$ for all $i=1,2,3$, where $I$ denotes the identity mapping, are demiclosed at zero, then $\left\{x_{n}\right\}$ converges weakly to a common fixed point of the mappings $T_{1}, T_{2}$ and $T_{3}$.

Proof. Let $q \in F$, from Lemma 3.1 the sequence $\left\{\left\|x_{n}-q\right\|\right\}$ is convergent and hence bounded. Since $E$ is uniformly convex, every bounded subset of $E$ is weakly compact. Thus there exists a subsequence $\left\{x_{n_{k}}\right\} \subset\left\{x_{n}\right\}$ such that $\left\{x_{n_{k}}\right\}$ converges weakly to $q^{*} \in C$. From Lemma 3.2, we have

$$
\lim _{k \rightarrow \infty}\left\|x_{n_{k}}-T_{i} x_{n_{k}}\right\|=0, \text { for all } i=1,2,3 .
$$

Since the mappings $I-T_{i}$ for all $i=1,2,3$ are demiclosed at zero, therefore $T_{i} q^{*}=q^{*}$ for all $i=1,2,3$, which means $q^{*} \in F$. Finally, let us prove that $\left\{x_{n}\right\}$ converges weakly to $q^{*}$. Suppose on contrary that there is a subsequence $\left\{x_{n_{j}}\right\} \subset\left\{x_{n}\right\}$ such that $\left\{x_{n_{j}}\right\}$ converges weakly to $p^{*} \in C$ and $q^{*} \neq p^{*}$. Then by the same method as given above, we can also prove that $p^{*} \in F$. From Lemma 3.1 the $\operatorname{limits}_{\lim _{n \rightarrow \infty}}\left\|x_{n}-q^{*}\right\|$ and $\lim _{n \rightarrow \infty}\left\|x_{n}-p^{*}\right\|$ exist. By virtue of the Opial condition of $E$, we obtain

$$
\begin{aligned}
\lim _{n \rightarrow \infty}\left\|x_{n}-q^{*}\right\| & =\lim _{n_{k} \rightarrow \infty}\left\|x_{n_{k}}-q^{*}\right\| \\
& <\lim _{n_{k} \rightarrow \infty}\left\|x_{n_{k}}-p^{*}\right\| \\
& =\lim _{n \rightarrow \infty}\left\|x_{n}-p^{*}\right\| \\
& =\lim _{n_{j} \rightarrow \infty}\left\|x_{n_{j}}-p^{*}\right\| \\
& <\lim _{n_{j} \rightarrow \infty}\left\|x_{n_{j}}-q^{*}\right\| \\
& =\lim _{n \rightarrow \infty}\left\|x_{n}-q^{*}\right\|
\end{aligned}
$$

which is a contradiction, so $q^{*}=p^{*}$. Thus $\left\{x_{n}\right\}$ converges weakly to a common fixed point of the mappings $T_{1}, T_{2}$ and $T_{3}$. This completes the proof.

Lemma 4.1. Under the conditions of Lemma 3.2 and for any $p, q \in F$, $\lim _{n \rightarrow \infty}\left\|t x_{n}+(1-t) p-q\right\|$ exists for all $t \in[0,1]$.

Proof. By Lemma 3.1, $\lim _{n \rightarrow \infty}\left\|x_{n}-q^{*}\right\|$ exists for all $q^{*} \in F$ and therefore $\left\{x_{n}\right\}$ is bounded. Letting

$$
a_{n}(t)=\left\|t x_{n}+(1-t) p-q\right\|
$$


for all $t \in[0,1]$. Then $\lim _{n \rightarrow \infty} a_{n}(0)=\|p-q\|$ and $\lim _{n \rightarrow \infty} a_{n}(1)=\left\|x_{n}-q\right\|$ exists by Lemma 3.1. It, therefore, remains to prove the Lemma 4.1 for $t \in(0,1)$. For all $x \in C$, we define the mapping $G_{n}: C \rightarrow C$ by:

$$
\begin{gathered}
A_{n}(x)=\left(1-\gamma_{n}\right) x+\gamma_{n} T_{3}^{n} x \\
B_{n}(x)=\left(1-\beta_{n}\right) A_{n}(x)+\beta_{n} T_{2}^{n} A_{n}(x)
\end{gathered}
$$

and

$$
G_{n}(x)=\left(1-\alpha_{n}\right) B_{n}(x)+\alpha_{n} T_{1}^{n} B_{n}(x) .
$$

Then it follows that $z_{n}=A_{n} x_{n}, y_{n}=B_{n} x_{n}, x_{n+1}=G_{n} x_{n}$ and $G_{n} p=p$ for all $p \in F$. Now from (21), (25) and (29) of Lemma 3.1, we see that

$$
\begin{aligned}
&\left\|A_{n}(x)-A_{n}(y)\right\| \leq\left(\frac{1+r_{n}+s_{n}}{1-s_{n}}\right)\|x-y\| \\
&\left\|B_{n}(x)-B_{n}(y)\right\| \leq\left(\frac{1+r_{n}+s_{n}}{1-s_{n}}\right)^{2}\|x-y\|
\end{aligned}
$$

and

$$
\begin{aligned}
\left\|G_{n}(x)-G_{n}(y)\right\| & \leq\left(1+Q h_{n}\right)\left\|x_{n}-q\right\| \\
& =l_{n}\|x-y\|,
\end{aligned}
$$

for some $Q>0$ and for all $x, y \in C$, where $l_{n}=1+Q h_{n}$ with $\sum_{n=1}^{\infty} h_{n}<\infty$ and $l_{n} \rightarrow 1$ as $n \rightarrow \infty$. Setting

$$
U_{n, m}=G_{n+m-1} G_{n+m-2} \ldots G_{n}, m \geq 1
$$

and

$$
b_{n, m}=\left\|U_{n, m}\left(t x_{n}+(1-t) p\right)-\left(t U_{n, m} x_{n}+(1-t) U_{n, m} q\right)\right\| .
$$

From (63) and (64), we have

$$
\begin{aligned}
& \left\|U_{n, m}(x)-U_{n, m}(y)\right\| \\
= & \left\|G_{n+m-1} G_{n+m-2} \ldots G_{n}(x)-G_{n+m-1} G_{n+m-2} \ldots G_{n}(y)\right\| \\
\leq & l_{n+m-1}\left\|G_{n+m-2} \ldots G_{n}(x)-G_{n+m-2} \ldots G_{n}(y)\right\| \\
\leq & l_{n+m-1} l_{n+m-2}\left\|G_{n+m-3} \ldots G_{n}(x)-G_{n+m-3} \ldots G_{n}(y)\right\| \\
& \vdots \\
\leq & \left(\prod_{j=n}^{n+m-1} l_{j}\right)\|x-y\| \\
(66) \leq & J_{n}\|x-y\|
\end{aligned}
$$

for all $x, y \in C$, where $J_{n}=\prod_{j=n}^{n+m-1} l_{j}$ and $U_{n, m} x_{n}=x_{n+m}, U_{n, m} p=p$ for all $p \in F$. Thus

$$
\begin{aligned}
a_{n+m}(t) & =\left\|t x_{n+m}+(1-t) p-q\right\| \\
& \leq b_{n, m}+\left\|U_{n, m}\left(t x_{n}+(1-t) p\right)-q\right\|
\end{aligned}
$$




$$
\leq b_{n, m}+J_{n} a_{n}(t)
$$

By using [ [3], Theorem 2.3], we have

$$
\begin{aligned}
b_{n, m} & \leq \varphi^{-1}\left(\left\|x_{n}-u\right\|-\left\|U_{n, m} x_{n}-U_{n, m} u\right\|\right) \\
& \leq \varphi^{-1}\left(\left\|x_{n}-u\right\|-\left\|x_{n+m}-u+u-U_{n, m} u\right\|\right) \\
& \leq \varphi^{-1}\left(\left\|x_{n}-u\right\|-\left(\left\|x_{n+m}-u\right\|-\left\|U_{n, m} u-u\right\|\right)\right)
\end{aligned}
$$

and so the sequence $\left\{b_{n, m}\right\}$ converges uniformly to 0 , i.e., $b_{n, m} \rightarrow 0$ as $n \rightarrow \infty$. Since $\lim _{n \rightarrow \infty} J_{n}=1$, therefore from (67), we have

$$
\limsup _{n \rightarrow \infty} a_{n}(t) \leq \lim _{n, m \rightarrow \infty} b_{n, m}+\liminf _{n \rightarrow \infty} a_{n}(t)=\liminf _{n \rightarrow \infty} a_{n}(t) .
$$

This shows that $\lim _{n \rightarrow \infty} a_{n}(t)$ exists, that is, $\lim _{n \rightarrow \infty}\left\|t x_{n}+(1-t) p-q\right\|$ exists for all $t \in[0,1]$. This completes the proof.

Theorem 4.2. Let $E$ be a real uniformly convex Banach space such that its dual $E^{*}$ has the Kadec-Klee property and $C$ be a nonempty closed convex subset of $E$. Let $T_{1}, T_{2}, T_{3}: C \rightarrow C$ be three uniformly L-Lipschitzian and generalized asymptotically quasi-nonexpansive mappings with sequences $\left\{r_{n}\right\}$ and $\left\{s_{n}\right\}$ as defined in proposition 2.1 such that $\sum_{n=1}^{\infty} \frac{r_{n}+2 s_{n}}{1-s_{n}}<\infty$ and $F=\cap_{i=1}^{3} F\left(T_{i}\right) \neq \emptyset$. Let $\left\{x_{n}\right\}$ be the iteration scheme defined by (12), where $\left\{\alpha_{n}\right\},\left\{\beta_{n}\right\}$ and $\left\{\gamma_{n}\right\}$ are sequences in $[\rho, 1-\rho]$ for all $n \in \mathbb{N}$ and for some $\rho \in(0,1)$. If the mappings $I-T_{i}$ for all $i=1,2,3$, where $I$ denotes the identity mapping, are demiclosed at zero, then $\left\{x_{n}\right\}$ converges weakly to a common fixed point of the mappings $S$ and $T$.

Proof. By Lemma 3.1, $\left\{x_{n}\right\}$ is bounded and since $E$ is reflexive, there exists a subsequence $\left\{x_{n_{j}}\right\}$ of $\left\{x_{n}\right\}$ which converges weakly to some $p \in C$. By Lemma 3.2, we have

$$
\lim _{j \rightarrow \infty}\left\|x_{n_{j}}-T_{i} x_{n_{j}}\right\|=0 \text { for all } 1,2,3 .
$$

Since by hypothesis the mappings $I-T_{i}$ for all $i=1,2,3$ are demiclosed at zero, therefore $T_{i} p=p$ for all $i=1,2,3$, which means $p \in F$. Now, we show that $\left\{x_{n}\right\}$ converges weakly to $p$. Suppose $\left\{x_{n_{i}}\right\}$ is another subsequence of $\left\{x_{n}\right\}$ converges weakly to some $q \in C$. By the same method as above, we have $q \in F$ and $p, q \in w_{w}\left(x_{n}\right)$. By Lemma 4.1, the limit

$$
\lim _{n \rightarrow \infty}\left\|t x_{n}+(1-t) p-q\right\|
$$

exists for all $t \in[0,1]$ and so $p=q$ by Lemma 2.3. Thus, the sequence $\left\{x_{n}\right\}$ converges weakly to $p \in F$. This completes the proof.

Example 4.1. Let $E=\mathbb{R}$ with the usual norm $|$.$| and C=[0, \infty)$. Let $T_{1}, T_{2}, T_{3}: E \rightarrow E$ be defined by $T_{1}(x)=\frac{x}{3}, T_{2}(x)=x \cos x$ and $T_{3}(x)=$ $\frac{x}{2} \sin x$. Then $T_{1}, T_{2}$ and $T_{3}$ are asymptotically quasi-nonexpansive mappings with constant sequence $\left\{k_{n}\right\}=\{1\}$. Hence by implication relation (7), they 
are generalized asymptotically quasi-nonexpansive mappings with common fixed point 0 , that is, $F=F\left(T_{1}\right) \cap F\left(T_{2}\right) \cap T_{3}=\{0\}$.

\section{CONCluding REMARKS}

In this paper, we establish some strong and weak convergence theorems of modified $S P$-iteration scheme for three generalized asymptotically quasinonexpansive mappings in the framework of uniformly convex Banach spaces. The said class of mappings is larger than the class of nonexpansive, quasinonexpansive, asymptotically nonexpansive and asymptotically quasi-nonexp ansive mappings. Thus the results presented in this paper extend, generalize and improve some known results from the current existing literature by means of spaces and iteration schemes considered in this paper.

\section{REFERENCES}

[1] R.P. Agarwal, Donal O'Regan, D.R. Sahu, Iterative construction of fixed points of nearly asymptotically nonexpansive mappings, Nonlinear Convex Anal. 8, No.1 (2007), 61-79.

[2] H. Fukhar-ud-din, S.H. Khan, Convergence of iterates with errors of asymptotically quasi-nonexpansive mappings and applications, J. Math. Anal. Appl. 328 (2007), 821-829.

[3] J. Garcia Falset, W. Kaczor, T. Kuczumow, S. Reich, Weak convergence theorems for asymptotically nonexpansive mappings and semigroups, Nonlinear Anal., TMA, 43, No.3 (2001), 377-401.

[4] K. Goebel, W.A. Kirk, A fixed point theorem for asymptotically nonexpansive mappings, Proc. Amer. Math. Soc. 35, No.1 (1972), 171-174.

[5] R. Glowinski, P. Le Tallec, "Augemented Lagrangian and Operator-Splitting Methods in Nonlinear Mechanics" Siam, Philadelphia, 1989.

[6] S. Haubruge, V.H. Nguyen, J.J. Strodiot, Convergence analysis and applications of the Glowinski Le Tallec splitting method for finding a zero of the sum of two maximal monotone operators, J. Optim. Theory Appl. 97 (1998), 645-673.

[7] S.H. Khan, Two-step iterative process for common fixed points of two asymptotically quasi-nonexpansive mappings, World Academy of Science, Engineering and Technology 75 (2011), 672-676.

[8] H.Y. Lan, Common fixed point iterative processes with errors for generalized asymptotically quasi-noexpansive mappings, Comput. Math. Appl. 52 (2006), 1403-1412.

[9] Q.H. Liu, Iterative sequences for asymptotically quasi-nonexpansive mappings, J. Math. Anal. Appl. 259 (2001), 1-7.

[10] N. Maiti, M.K. Ghosh, Approximating fixed points by Ishikawa iterates, Bull. Aust. Math. Soc. 40 (1989), 113-117.

[11] J. Nantadilok, Three-step iteration scheme with errors for generalized asymptotically quasi-nonexpansive mappings, Thai J. Math. 6, No.2 (2008), 297-308. 
[12] M.A. Noor, New approximation schemes for general variational inequalities, J. Math. Anal. Appl. 251, No.1 (2000), 217-229.

[13] M.A. Noor, Three-step iterative algorithms for multivalued quasi variational inclusions, J. Math. Anal. Appl. 255 (2001), 589-604.

[14] Z. Opial, Weak convergence of the sequence of successive approximations for nonexpansive mappings, Bull. Amer. Math. Soc. 73 (1967), 591-597.

[15] W. Phuengrattana, S. Suantai, On the rate of convergence of Mann, Ishikawa, Noor and $S P$ iterations for continuous functions on an arbitrary interval, J. Comput. Appl. Math. 235 (2011), 3006-3014.

[16] G.S. Saluja, Strong convergence theorem for two asymptotically quasi-nonexpansive mappings with errors in Banach space, Tamkang J. Math. 38, No.1 (2007), 85-92.

[17] G.S. Saluja, Convergence theorems of modified two-step iteration process for two asymptotically quasi-nonexpansive mappings, Adv. Fixed Point Theory 3, No.1 (2013), 174-194.

[18] G.S. Saluja, J.K. Kim, Convergence of common fixed point of finite step iteration process for generalized asymptotically quasi-nonexpansive mappings, Nonlinear Funct. Anal. Appl. 19, No.1 (2014), 79-98.

[19] G.S. Saluja, H.K. Nashine, Weak convergence theorems of two-step iteration process for two asymptotically quasi-nonexpansive mappings, Indian J. Maths 56, No.3 (2014), 291-311.

[20] G.S. Saluja, Convergence theorems for two finite families of asymptotically quasinonexpansive mappings in Banach spaces, Romai J. 10, No.1 (2014), 157-173.

[21] J. Schu, Weak and strong convergence to fixed points of asymptotically nonexpansive mappings, Bull. Austral. Math. Soc. 43, No.1 (1991), 153-159.

[22] H.F. Senter, W.G. Dotson, Approximating fixed points of nonexpansive mappings, Proc. Amer. Math. Soc. 44 (1974), 375-380.

[23] N. Shahzad, A. Udomene, Approximating common fixed points of two asymptotically quasi-nonexpansive mappings in Banach spaces, Fixed Point Theory and Applications, Vol. 2006, Article ID 18909, Pages 1-10.

[24] K. Sitthikul, S. Saejung, Convergence theorems for a finite family of nonexpansive and asymptotically nonexpansive mappings, Acta Univ. Palack. Olomuc. Math. 48 (2009), 139-152.

[25] K.K. Tan, H.K. Xu, Approximating fixed points of nonexpansive mappings by the Ishikawa iteration process, J. Math. Anal. Appl. 178 (1993), 301-308.

[26] B.L. Xu, M.A. Noor, Fixed point iterations for asymptotically nonexpansive mappings in Banach spaces, J. Math. Anal. Appl. 267 (2002), 444-453.

\author{
G.S. Saluja \\ Department of Mathematics \\ Govt. N.P.G. College of Science \\ RAIPUR - 492001 (C.G.) \\ INDIA \\ E-mail address: saluja1963@gmail.com
}

